\title{
LA SALUD: UN CONSUMO PRIVILEGIADO
}

\section{HEALTH: A PRIVILEGED CONSUMPTION}

\author{
Ignacio López Seco ${ }^{1}$
}

DOI: https://doi.org/10.37767/2591-3476(2021)15

\author{
Comentario a \\ W.,D. c/ OMINT S.A. DE SERVICIOS s/ incumplimiento de prestación de \\ obra social/med. prepaga. \\ Cámara Civil y Comercial Federal, Sala I - Poder Judicial de la Nación
}

\author{
Disponible en \\ https://bit.ly/38gllbk
}

\section{RESUMEN:}

El presente comentario aborda el razonamiento formulado por la Cámara Civil y Comercial Federal del Poder Judicial de la Nación, que confirmó la sentencia de primera instancia, que acogió la pretensión del actor, condenado a la demandada, entidad de medicina prepaga, a disponer el cambio de plan médico que fuera unilateralmente denegado por ésta, así como también, al reintegro de los gastos que el accionante desembolsó, como consecuencia de dicha negativa. En el presente caso, el actor transitaba una enfermedad oncológica, que motivó la solicitud del cambio del plan contratado, lo que fue resistido por la entidad de medicina prepaga. Ello motivó que, frente al avance de su enfermedad, y la negativa de la demandada a efectivizar el cambio de plan, el actor desembolse, de manera particular, el importe correspondiente al tratamiento que el plan contratado no cubría. La demandada, resistió la pretensión, alegando que no está obligada a asegurar el derecho a la salud. El razonamiento reproducido por la sentencia de Cámara descansa en dos aspectos fundamentales: por un lado, la imposibilidad de que la demandada sea desvinculada de la salud de su afiliado, y por el otro lado, la aplicación del régimen tuitivo consumeril al contrato de medicina prepaga.

\section{ABSTRACT}

This comment contains the reasoning formulated by the Federal Civil and Commercial Chamber of the Judicial Power of the Nation, which confirmed the sentence of first instance, which accepted the claim of the plaintiff, sentenced to the defendant, a prepaid medicine entity, to order the change of the medical plan that was unilaterally denied by it, as well as the

1 Abogado (Universidad Nacional de Córdoba); Martillero y Corredor Público (Colegio Nacional de Monserrat); Maestrando en Derecho Procesal (Universidad Siglo XXI). Se desempeña como Auxiliar en el Juzgado Civil y Comercial de $1^{\circ}$ Instancia y $36^{\circ}$ Nominación. Mail: ignaciolopezsec@hotmail.com. ORCID iD: https://orcid.org/0000-0002-2582-5856 
reimbursement of the expenses that the plaintiff disbursed. In the present case, the plaintiff was suffering from an oncological disease, which led to the request to change the contracted plan, which was resisted by the prepaid medicine entity. This led to the fact that, given the advance of his illness, and the defendant's refusal to effect the change of medical plan, the plaintiff paid, the amount corresponding to the treatment that the contracted plan did not cover. The defendant resisted the claim, claiming that it is not obliged to ensure the right to health. The reasoning reproduced by the Chamber rests on two fundamental aspects: in firs place, the impossibility for the defendant to be disconnected from the health of its affiliate, in second place, the application of the consumer protection regime to the prepaid medicine contract.

PALABRAS CLAVE: Medicina prepaga; Derecho a la salud; Defensa del consumidor; Abuso del derecho; Vulnerabilidad.

KEY WORDS: Prepaid medicine; Right to health; Consumer Defense; Abuse of rights; Vulnerability.

\section{Introducción}

En el mes de mayo del año 2020, en los autos caratulados "W.,D. c/ OMINT S.A. DE SERVICIOS S/ INCUMPLIMIENTO DE PRESTACIÓN DE OBRA SOCIAL/MED. PREPAGA", Ia Cámara Civil y Comercial Federal, Sala I, del Poder Judicial de la Nación, resolvió confirmar a sentencia de primera instancia dictada con fecha 25/10/2019 por el magistrado a cargo del Juzgado Civil Y Comercial Federal N. ${ }^{\circ} 4$. En esta última resolución, el magistrado de primera instancia resolvió condenar a la demandada, Omint S.A. de Servicios, entidad de medicina prepaga, a disponer el cambio de plan médico que fuera unilateralmente denegado por ésta al accionante, así como también, al reintegro de los gastos que el pretensor desembolsó, como consecuencia de dicha negativa.

\section{I) Los hechos.}

Conforme surge de la resolución de primera instancia, confirmada por la sentencia comentada, el actor, D.W, promueve demanda en contra de Omint S.A de Servicios a fin de que disponga el cambio de plan de asistencia médica que le fue negado por la demandada en sede extrajudicial, así como también el reintegro de los gastos que incurrió como consecuencia de dicha negativa, con más daños y perjuicios.

El accionante relata que en el año 2013 se realizó una biopsia en el Centro de Urología y el resultado fue positivo, indicando la existencia de un adenocarcinoma. Frente a tal panorama, el accionante, Sr. D.W, realiza una interconsulta con el Jefe de Servicio de Urología del Hospital Alemán, quien forma parte integrante de la cartilla de Omint, pero no pertenece a los prestadores de la cartilla que el actor había contratado.

En consecuencia, con fecha 26/12/2013, el actor concurre a la prestadora Omint, para solicitar el cambio de plan contratado, quien rechaza su solicitud.

Luego de ello, el Sr. D.W, ante la apremiante urgencia de su enfermedad, comenzó el tratamiento con el jefe de Urología del Hospital Alemán, quien le recomendó la reali- 
zación de una intervención quirúrgica, debiendo afrontar los gastos de esta. Asimismo, luego de la intervención, los médicos le indicaron un tratamiento protocolar de "rayos de intensidad media modulada", en el centro médico "VIDT", prestador de Omint, pero perteneciente a un plan distinto al que el afiliado había originalmente contratado.

El Actor señala que al requerir a la demandada la cobertura del tratamiento, esta se la negó alegando que a su criterio bastaban los rayos 3D. Frente a esta denegación, el accionante asumió de manera particular el costo de la prestación.

Por su parte, la demandada, resiste la pretensión del accionante, alegando que su afiliado, el Sr. D.W contrató con la entidad un plan cerrado, por lo que, si el afiliado decide acudir a médicos y centros asistenciales de la cartilla de su mandante, la cobertura no tiene ningún tipo de límite o tope, sin embargo, si decide acudir a prestadores ajenos, su mandante no tiene obligación contractual de afrontar los gastos generados.

Alegó también que las solicitudes de cambio de plan se encuentran sujetas al análisis y aprobación de la entidad de medicina prepaga.

Finalmente, señaló que el actor, solicitó el cambio de plan, fuera del plazo predeterminado por la empresa, esto es, del 1 al 20 de abril de cada año, conforme a la cláusula 4.3 del Reglamento de Servicios que es parte integrante del contrato que vincula a la empresa demandada con sus afiliados.

\section{I) El fallo de primera instancia.}

En su resolución, el magistrado de primera instancia comienza sentando los criterios jurisprudenciales de la Corte Suprema de Justicia de la Nación, vinculados al derecho de la Salud. En tal sentido, expone que el derecho a la vida es el primer derecho de la persona humana que resulta reconocido y garantizado por la Constitución Nacional.

Expresa que nuestro máximo tribunal nacional, reafirmó que el ordenamiento convencional, con jerarquía constitucional (art. 75 inciso $22 \mathrm{CN}$ ), establece que el derecho a la Salud, comprendido dentro del derecho a la vida, conlleva la obligación impostergable que debe desarrollar la autoridad publica para garantizarlo con acciones positivas, sin perjuicio de las obligaciones que deban asumir las jurisdicciones locales, las obras sociales o las entidades llamadas de medicina prepaga. De allí que el Pacto Internacional de Derechos Económicos, Sociales y Culturales reconoce el derecho que se le otorga a todas las personas a disfrutar del más alto nivel posible de salud física y mental (art.12.1). Asimismo, refiere que las empresas de medicina prepaga se encuentran igualadas en cuanto a sus obligaciones prestacionales en relación con las obras sociales (confr. CNCCFed., Sala I, causa 3054/2013 del 3.3.2013 y 3959/18 del 7.2.2019, entre otras).

Luego de reseñada la jurisprudencia de la Corte Suprema de Justicia de la Nación, el magistrado de primera instancia, señala que resulta aplicable el régimen tuitivo del derecho consumeril al contrato de cobertura médica celebrado entre las partes y atento que los usuarios y consumidores son sujetos particularmente vulnerables, la interpretación de las cláusulas y prerrogativas insertas en el contrato o reglamento deberá, ajustarse a los principios del derecho de consumo, como la buena fe, el trato digno, y no discriminación, el deber de información adecuada y veráz y en caso de duda, la interpretación mas favorable para el consumidor (art. 42 de la Constitución Nacional y art. 37 de la ley 24.240). 
El magistrado, señala que no se puede prescindir de la función social que tiene el contrato de medicina prepaga, en virtud de los bienes en juego, como la salud y la vida de las personas, protegidos por la Constitución Nacional y tratados internacionales, máxime cuando ningún ordenamiento jurídico puede justificar el abuso del derecho.

La sentencia de primera instancia resuelve que le asiste derecho al actor al cambio de plan médico asistencial a un plan superador con relación al originalmente contratado, y por el otro, le asiste derecho al reintegro de los gastos afrontados de manera particular, como consecuencia de la negativa de la demandada a la pretensión del actor.

\section{II) La sentencia de Cámara.}

Contra la sentencia de primera instancia, la demandada Omint se alza en apelación. Se agravia, en cuanto alega que no es su parte obligada a asegurarle la contraria el derecho a la salud, ya que se trata de una sociedad anónima que no ha asumido tal obligación, bajo ningún concepto, al mismo tiempo que aduce, que es el Estado Nacional, es quien ostenta en general, el carácter de garante de la salud de la población. Invoca que no está obligada a cubrir una prestación médica más allá de lo convenido con los accionantes y que la negativa al cambio de plan solicitado constituye un acto discrecional de la empresa, facultad que ha ejercido en forma razonable.

Tal como se adelantó, esta resolución rechaza los agravios de la demandada y confirma la resolución de primera instancia.

Para decidir de tal manera, en primer lugar, la Cámara hizo hincapié en que, conforme a la jurisprudencia de la Corte Suprema de Justicia de la Nación, la obligación del Estado Nacional de garantizar el derecho a la salud con acciones positivas, lo es sin perjuicio de las obligaciones que deban asumir en su cumplimiento las entidades de medicina prepaga (conf. Fallos 328:4640; entre muchos otros).

En este sentido, este agravio resulta inadmnisible, toda vez que la obligación principal de la empresa de medicina prepaga, en el sistema de cartilla o sistema cerrado, es justamente prestar el servicio médico, lo que lleva a cabo a través de los integrantes de la cartilla.

La sentencia, refiere sobre ello que: "En base a ese estándar, la predicada ajenidad de la demandada respecto de la salud de los actores, no resulta un argumento atendible. Máxime, si se pondera que la prestación de servicios de salud es la actividad comercial a la que se dedica."

Luego, continúa su razonamiento, al señalar que la controversia no se circunscribe a establecer si corresponde obligar a la demandada a asumir una prestación médica no convenida contractualmente, sino de dirimir si su negativa a encuadrar a los actores en un plan superador al que habían contratado fue legítima.

Al respecto, concluye que la cláusula 4.3 del Reglamento de Servicios, que es parte integrante del contrato que vincula a la empresa demandada con sus afiliados y establece la posibilidad de que estos manifiesten sus intenciones de cambio de plan una vez al año, entre el 1 y el 20 de abril, debe ser interpretada a la luz de los principios del régimen de defensa del consumidor, plenamente aplicable al contrato de medicina prepaga. A la luz 
de tales principios, la resolución resuelve que, estando en juego el derecho a la salud de las personas, la denegatoria del cambio de plan formulado por la demandada constituye un abuso del derecho.

La resolución destaca que, conforme la Jurisprudencia de nuestro máximo tribunal nacional, el régimen de defensa del consumidor es plenamente aplicable al contrato de medicina prepaga por lo que la interpretación de las cláusulas y prerrogativas del contrato deben efectuarse de acuerdo con la normativa consumeril. En este entendimiento, señala que la negativa de la demandada a concretar el cambio de plan del accionante, con fundamento en que este no efectuó en el periodo habilitado a tales efectos (del 1 al 20 de abril, conforme la cláusula 4.3 del Reglamento de Servicios), configura un abuso del derecho, máxime, cuando está en juego el derecho a la salud de las personas.

Respecto de esto último, la sentencia de Cámara agrega que el abuso del derecho se configura en el presente caso, en tanto la demandada no ha brindado ninguna razón atendible que justifique la negativa a brindar mayor cobertura, asumiendo el actor el costo que representa el nuevo plan, lo que se agrava en función del delicado estado de salud que transitaba el accionante.

\section{III) El derecho a la salud como punto de partida.}

Se advierte que, en ambas resoluciones, esto es, tanto en la sentencia de Cámara comentada, así como también en la sentencia de primera instancia que ésta confirma, el razonamiento de los magistrados inicia abordando el derecho a la salud, inescindiblemente vinculado al derecho a la vida y a la dignidad humana.

En este sentido, el punto de partida del razonamiento es impecable, puesto que logra encuadrar la cuestión debatida, para luego, dentro de ese enfoque (insisto, el derecho a la salud, la vida y la dignidad humana), aportar herramientas conceptuales, que integran un sistema más amplio, de protección de derechos reconocidos en el orden constitucional y convencional.

El fin último del ordenamiento legal (en sentido lato), su razón de ser, es la persona humana. Esta premisa implica un sistema de ponderación de los bines jurídicos reconocidos, dentro del cual, el primer derecho que debe ser reconocido y tutelado, es el derecho a la vida y la integridad psicofísica de las personas. Esto toda vez que, el resto de los derechos y garantías consagrados en el ordenamiento positivo, se encuentran subordinados al derecho a la vida, presupuesto ineludible para el ejercicio de los demás.

En nuestra Constitución Nacional, el derecho a la salud ha sido introducido en la reforma del año 1994, con la incorporación de los tratados de derechos internacionales de derechos humanos, que constituyen el llamado bloque de constitucionalidad (art. 75 inciso $\left.22^{\circ} \mathrm{CN}\right)$.

Previo a ello, con frecuencia la doctrina ubicaba el derecho a la salud en el marco de los derechos implícitos del art. 33 de la Constitución Nacional. ${ }^{2}$

Asimismo, debe destacarse que, a partir del año 2015, con la reforma del Código Civil y

2 Diaz Ana Inés (2019). “Conceptualización del derecho a la salud en la Argentina” -. Publicado en: LA LEY 29/10/2019, 1 • LA LEY 2019-E , 1135 - Cita: TR LALEY AR/DOC/2805/2019. 
Comercial de la Nación se acogió normativamente la constitucionalización del derecho privado (art. $1^{\circ} \mathrm{CCCN}$ ).

Ahora bien, en cuanto al ordenamiento convencional, con jerarquía constitucional (art. 75 inciso $22^{\circ} \mathrm{CN}$ ) vinculado al derecho a la salud, se destacan: la Declaración Americana de los Derechos y Deberes del Hombre (Aprobada en Bogotá en el año 1948), cuyo artículo 11 dispone: "Toda persona tiene derecho a que su salud sea preservada por medidas sanitarias y sociales, relativas a la alimentación, el vestido, la vivienda y la asistencia médica, correspondientes al nivel que permitan los recursos públicos y los de la comunidad." Encontramos también el Pacto Internacional de Derechos Económicos Sociales y Culturales, cuyo artículo 12, inciso $1^{\circ}$ reza: "Los Estados Partes en el presente Pacto reconocen el derecho de toda persona al disfrute del más alto nivel posible de salud física y mental." En la Convención Americana sobre Derechos Humanos, también conocida como Pacto de San José de Costa Rica, en el art. 4 inciso $1^{\circ}$ se reconoce el derecho a la vida: "toda persona tiene derecho a que se respete su vida" y en su art. 5 el Derecho a la integridad personal, disponiendo el inciso $1^{\circ}$ que "Toda persona tiene derecho a que se respete su integridad física, psíquica y moral". La Declaración Universal de Derechos Humanos, en su art. 25 inciso $1^{\circ}$ dispone: "Toda persona tiene derecho a un nivel de vida adecuado que le asegure, así como a su familia, la salud y el bienestar, y en especial la alimentación, el vestido, la vivienda, la asistencia médica y los servicios sociales necesarios (...)"

De la normativa citada, resulta innegable la prevalencia de ciertas normas y principios con las que el ordenamiento positivo tutela el derecho a la vida y a la salud, como bien jurídico protegido, y es a la luz de tales principios que la resolución que la sentencia de Cámara advierte como cuestión liminar, que deberá resolverse el caso traído a su conocimiento.

Ahora bien, considero, que lo enriquecedor de la resolución comentada, recae en el hecho que la Cámara no se detiene solo solo en este encuadre jurídico, sino que da un paso más, e integra, con acierto, estos principios a una rama del derecho, que igualmente tutela a las personas, pero frente a determinados tipos de abusos, no necesariamente vinculados al derecho a la salud: el régimen de defensa del consumidor.

\section{IV) El derecho a la salud y el derecho de consumo. Dialogo de fuentes.}

Recapitulado, la sentencia comentada, encuadra el caso en el régimen protectorio del derecho a la vida, y dentro de ello, a la salud del afiliado de la entidad de medicina prepaga, en base al ordenamiento convencional (con jerarquía constitucional), al que se hizo referencia en el punto anterior.

A ese régimen, protectorio de la persona en su integridad psicofísica, y que encuentra su fundamento en el valor de la vida humana, la resolución lo integra, de manera armónica, con el derecho de defensa del consumidor. En tal sentido, la Cámara señala que la Corte Suprema de Justicia de la Nación establece que el régimen de defensa del consumidor es plenamente aplicable al contrato de medicina prepaga, lo que conlleva a que la interpretación de las cláusulas y prerrogativas insertas en el contrato o reglamento deberá efectuarse con arreglo a la normativa consumeril. (del dictamen del procurador general que la Corte hace suyo) (*13/03/2001*, E., R. E. c/ Omint S.A. de Servicios*, LA LEY 2001-B, 678 -L LEY 2001-E, 22, con nota de Inés A. D’Argento -DJ 2001-2, 87- ED del 09/05/2001, p. 9) (confr. CNCCFed. Civil y Comercial, Sala II, causa 45.393/95 del 20/12/05).

Sobre esto último, la doctrina advertía: "De esta forma, la jurisprudencia enroló los contra- 
tos de medicina prepaga bajo las características expuestas de adhesión y consumo, atribuyéndoles las consecuencias jurídicas correspondientes y diferenciándolo de aquellos convenios en los cuales prima la paridad e igualdad contractual reconocida en los arts. 1137 y 1197, entre otros, del Código Civil (ley 340). ${ }^{\text {3 }}$

La sentencia de primera instancia, también señala que el contrato que vincula al actor, como afiliado del plan de la entidad de medicina prepaga demandada, es un típico contrato de adhesión, en el que las cláusulas son predispuestas unilateralmente por la accionada y el adherente solo cuenta con la posibilidad de aceptarlo o rechazarlo, sin posibilidad de discutir el contenido del negocio jurídico, que se presenta como inmodificable.

Ahora bien, esta posibilidad de integrar ambos regímenes normativos, ensanchando considerablemente la protección de los afiliados al régimen de medicina prepaga, requiere de un método de interpretación orgánico, comúnmente denominado en la doctrina como "dialogo de fuentes".

Esto permite la coordinación de las normas al interior del sistema jurídico, que deja atrás el modelo de las antinomias del derecho, que se basaba en que una regla debe excluir a otra por razones temporales o de especialidad. Así, en el dialogo de fuentes, la finalidad de las normas se da mediante el "dialogo entre ellas", lo que permite integrar el sistema, de manera coherente y coordinada.

Esta comunicación entre diversos regímenes jurídicos, puede presentarse de manera horizontal, es decir, entre nomas de igual jerarquía, de manera vertical, cuando la jerarquía no es la misma (por ejemplo entre un precepto legal y otro constitucional o convencional).

Es este sentido, cierta doctrina, respecto al "dialogo de fuentes", ha sostenido: "Esto no es una cuestión exclusiva en materia de Derecho del Consumidor, sino que esas "relaciones dialógicas" entre Código Civil y Comercial y leyes especiales se observa también en otras materias, como niñez y adolescencia, salud mental, personas jurídicas, ambiente, entre otras. Así, necesariamente para entender el dispositivo jurídico aplicable a un hecho social, requerirá necesariamente, no sólo de un "diálogo horizontal" entre el Código Civil y Comercial y las leyes especiales -en este caso, las leyes de protección al consumidor — sino también, previa, y más importante, un "diálogo vertical" de estas normas con aquellas de fuente constitucional y convencional. En este último caso no hay, en rigor, un "diálogo", pero sí un "monólogo", porque las reglas superiores preponderan necesariamente sobre aquellas que se sitúan en un nivel inferior". 4

Repárese, en que la reforma constitucional del año 1994 otorgó jerarquía constitucional al derecho de los consumidores, en el artículo 42, que dispone: "los consumidores y usuarios de bienes y servicios tienen derecho, en la relación de consumo, a la protección de su salud, seguridad e intereses económicos; a una información adecuada y veraz; a la libertad de elección y a condiciones de trato equitativo y digno", otorgando de tal modo jerarquía constitucional al principio protectorio del usuario o consumidor. ${ }^{5}$

3 Cao Christian Alberto (2016) - "La protección del derecho constitucional a la salud en los contratos de adhesión y consumo. Nota particular sobre el contrato de medicina prepaga, su impacto en el código civil y comercial y normas complementarias" - Publicado en: RCCyC 2016 (noviembre), 227 Cita: TR LALEY AR/ DOC/3189/2016)

4 Barocelli, Sergio Sebastián (2015), "Contratos de seguros y derecho del consumidor. Diálogo de fuentes y perspectivas a la luz del nuevo código civil y comercial”. Publicado en: rdco273, 1021, cita tr laley ar/doc/5192/2015.

5 (Cfr. CARLOS A. HERNÁNDEZ, SANDRA A. FRUSTAGLI (2003) JURISPRUDENCIA ARGENTINA-LEXIS NEXIS 2003-IV-1541 Id SAIJ: DASF070020) 
Terminan de integrar el régimen normativo de defensa del consumidor, la ley 24.240 con sus respectivas modificaciones y los artículos 1092 al 1122 del CCCN.

\section{v) La salud como consumo privilegiado.}

Sentadas estas bases, se advierte que, este sistema dialógico, integrado por el derecho a la salud y el derecho de consumo, tiene a la persona humana, adherente a un sistema de medicina prepaga mediante clausulas predispuestas, como centro de protección.

Autorizada doctrina conceptualiza al derecho del consumidor, como "un sistema global de normas, principios, instituciones e instrumentos de implementación, consagrados por el ordenamiento jurídico en favor del consumidor, para garantizarle en el mercado una posición de equilibrio en sus relaciones con los empresarios."

Asimismo, en cuanto a la aplicación del régimen del derecho del consumidor al contrato de medicina prepaga se ha sostenido "Sabido es que el contrato de medicina prepaga es fuente de derechos y deberes recíprocos entre la empresa y el usuario. Así como la primera, tiene la obligación de informar -en los términos del art. $4^{\circ}$ de la ley 24.240 y concordantes-; de cumplir las prestaciones de salud comprometidas; no interrumpir la cobertura sin causa justificada -continuidad y/o regularidad del servicio-; no rescindir unilateralmente el contrato sin causa justificada, garantizar condiciones de atención y trato digno (art. $8^{\circ}$ bis, LDC); etc. El adherente también debe informar (su declaración jurada debe ser completa y veraz para no incurrir en reticencia o falsedad); abonar las cuotas, prestar colaboración; abstenerse de hacer un uso indebido del sistema". ${ }^{7}$

En este entendimiento, los principios que orbitan el derecho de consumo, entre los que encontramos el principio protectorio, el orden público, in dubio consumidor, trato digno y equitativo, gratuidad, deber de información, obligación de seguridad y el principio de sustentabilidad, adquieren una dimensión particular, cuando se encuentra comprometida la salud del consumidor.

Esto último se justifica, en un aspecto al que hace referencia el fallo de primera instancia (al que la sentencia comentada se remite): la mayor vulnerabilidad de aquel sujeto que ve comprometida su salud. Ello justifica, que los principios tutelares del régimen consumeril, graviten incluso con mayor intensidad que si se tratase de un acto de consumo en el que no se ve directamente comprometida la salud del consumidor, como por ejemplo la contratación de un plan de telefonía celular, o la adquisición de un electrodoméstico.

Repárese que, en fallo comentado, el accionante sufría una grave enfermedad oncológica, que comprometía su gravemente su salud y ponía en peligro su vida. En tal sentido, la conducta desplegada por la demandada, consistente en la negativa de efectivizar el cambio de plan contratado por el afiliado, se agrava, en torno al desamparo en que la enfermedad puso al accionante, que se encontraba, sin hesitación, en situación de vulnerabilidad.

En este sentido, autorizada doctrina ha sostenido: "Si se analiza la definición de "persona vulnerable" desde el punto de vista del derecho a la salud, por ejemplo, podremos afirmar

6 Stiglitz Gabriel A. Ley de Defensa del Consumidor (una primera visión de conjunto) Publicado en: Jurisprudencia Argentina. Cita TR la ley 0003/011878

7 Nager María Agustina (2019) “Protección del consumidor de servicios médicos prepagos” Publicado en: RCCyC 2019 (julio) , 194 - Cita: TR LALEY AR/ DOC/1649/2019 
claramente que cualquier persona que necesite una resolución judicial por una afección en su salud es, a estos efectos, un sujeto vulnerable. El paciente siempre es vulnerable, tanto por no gozar plenamente de su estado de plenitud, como también por su engranaje dentro del sistema sanitario." (el énfasis me pertenece). ${ }^{8}$

Destaco que, la asimetría existente entre la entidad de medicina prepaga como proveedora de servicio de salud y el afiliado que pretende cambiar su plan de cobertura a uno superador (y contratado mediante un pliego de adhesión cláusulas preimpresas), se agrava con la condición de hipervulnerabilidad que transitaba este último. Ello, implica que los principios del derecho del consumidor, cuya finalidad descansa en contrarrestar los disvaliosos efectos que acarrea la posición asimétrica en la relación de consumo, operen, en casos donde se ve comprometida la integridad psicofísica del consumidor, en el máximo grado de protección posible.

En tal entendimiento, se destaca que las relaciones de consumo en las que se encuentra comprometido el derecho a la salud de las personas, y en base a la condición de particular vulnerabilidad que estas transitan, deben privilegiarse por sobre aquellas en donde no existe afección a la salud, y su protección debe ser necesariamente mayor. Esto, como una consecuencia natural del enfoque que pone en el centro del ordenamiento jurídico a la persona humana, y como principal bien jurídico protegido la vida y la salud.

Asi, la Cámara, resolvió el caso haciendo aplicación del régimen de defensa del consumidor al contrato de medicina prepaga, y concluyó que a la luz de los principios que orbitan en dicho régimen protectorio principio (protectorio, orden público, in dubio pro-consumidor, trato digno y equitativo, gratuidad, deber de información, obligación de seguridad y principio de sustentabilidad) resulta abusiva la postura de la entidad demandada, en cuanto se negó a realizar el cambio de plan solicitado a uno superador, sin justificar de ninguna forma dicha negativa.

\section{VI) CONCLUSION}

Entiendo que el valor del fallo comentado descansa en que logra, tal como se desarrolló a lo largo del presente trabajo, integrar diversos regímenes protectorios para abarcar una determinada relación jurídica, como lo es el contrato de medicina prepaga, que sin lugar a duda cumple una función de innegable trascendencia social.

Respecto a esto último, la resolución, señala con acierto que, siendo que la prestación de servicios de salud es justamente la actividad comercial a la que se dedica la entidad de medicina prepaga, esta no puede seriamente alegar ser ajena a la salud de sus afiliados.

Entiendo, que este primer abordaje que realiza la Cámara se vincula de manera directa con la función social del contrato de medicina prepaga, que justifica que las mismas tengan la obligación (sin perjuicio de la obligación que corresponda al Estado Nacional) de garantizar el derecho a la Salud.

Comparto plenamente este criterio, toda vez que implica reconocer el valor social de las actividades que realizan estas entidades, y establecer que las mismas están obligadas a prestar un servicio que por lo menos garantice el acceso a la salud de sus afialos.

8 Gonzalo Gabriel Carranza y Claudia Elizabeth Zalazar (2015) - "Justicia y personas en condición de vulnerabilidad” Revista Derecho y Salud | Universidad Blas Pascal 2018-11-15 | journal-article DOI: https://doi.org/10.37767/2591-3476(2018)01 
El derecho es una construcción social, cuyo fundamento es la persona humana. El derecho a la vida, es un derecho humano básico, y debe ser el primero en ser reconocido, puesto que es el presupuesto ineludible para el ejercicio de los demás derechos. En este entendimiento, la vida y la salud no pueden quedar librados exclusivamente a las condiciones del mercado, ni naufragar en su lógica implacable: la rentabilidad.

Por otra parte, el valor de la resolución comentada reside en la aplicación del régimen del consumidor a los contratos de medicina prepaga, integrando, junto al derecho a la vida y la salud, un sistema mucho más amplio de protección.

De esta manera, se reconoce a un sujeto que se encuentra en una situación doblemente desventajosa: por un lado, con fundamento en las relaciones asimétricas propias que se establecen entres proveedores y consumidores, y por el otro, por la vulnerabilidad en que lo coloca su afección a la salud. Frente a ello, se integra un sistema que reconoce esta realidad y otorga a este sujeto un marco tutelar propio, acorde a su situación de desamparo.

En definitiva, la idea de un "consumo privilegiado", busca de alguna manera sintetizar este régimen integrado por el derecho a la salud y el derecho al consumo (dialogo de fuentes) cuya premisa principal, residen en que, a mayor vulnerabilidad, mayor debe ser la protección que brinde el derecho.

\section{REFERENCIAS BIBLIOGRAFICAS:}

- Barocelli, Sergio Sebastián (2015), "Contratos de seguros y derecho del consumidor. Diálogo de fuentes y perspectivas a la luz del nuevo código civil y comercial". Publicado en: rdco273, 1021, cita tr laley ar/doc/5192/2015

- Cao, Christian Alberto (2016) - “La protección del derecho constitucional a la salud en los contratos de adhesión y consumo. Nota particular sobre el contrato de medicina prepaga, su impacto en el código civil y comercial y normas complementarias" - Publicado en: RCCyC 2016 (noviembre), 227 Cita: TR LALEY AR/DOC/3189/2016)

- Carlos a. Hernández, sandra a. Frustagli (2003)" jurisprudencia argentina"-lexis nexis 2003-iv-1541 id saij: dasf070020

- Diaz Ana Inés (2019). “Conceptualización del derecho a la salud en la argentina” -. Publicado en: LA LEY 29/10/2019, 1 • LA LEY 2019-E , 1135 - Cita: TR LALEY AR/DOC/2805/2019

- Gonzalo Gabriel Carranza y Claudia Elizabeth Zalazar (2015) - “Justicia y personas en condición de vulnerabilidad" Revista Derecho y Salud | Universidad Blas Pascal 2018-1115 | journal-article DOI: https://doi.org/10.37767/2591-3476(2018)01

- Nager María Agustina (2019) “Protección del consumidor de servicios médicos prepagos" Publicado en: RCCyC 2019 (julio) , 194 - Cita: TR LALEY AR/DOC/1649/2019

- Stiglitz Gabriel A. "Ley de Defensa del Consumidor (una primera visión de conjunto)" 